\title{
Excitation spectra and ground-state properties from density-functional theory for the inverted band-structure systems $\beta$-HgS, HgSe, and HgTe
}

\author{
A. Delin \\ Applied Materials Physics, Department of Materials Science and Engineering, KTH, Brinellvägen 23, 10044 Stockholm, Sweden \\ and ICTP, Strada Costiera 11, P.O. Box 586, 34100 Trieste, Italy \\ T. Klüner \\ Fritz-Haber-Institut der Max-Planck-Gesellschaft, Faradayweg 4-6, D-14195 Berlin, Germany
}

(Received 13 February 2002; published 29 July 2002)

\begin{abstract}
We have performed a systematic density-functional study of the mercury chalcogenide compounds $\beta$ - $\mathrm{HgS}$, $\mathrm{HgSe}$, and $\mathrm{HgTe}$ using an all-electron full-potential linear muffin-tin orbital method. We find that, in the zinc-blende structure, both $\mathrm{HgSe}$ and $\mathrm{HgTe}$ are semimetals whereas $\beta$-HgS has a small spin-orbit-induced band gap. Our calculated relativistic photoemission and inverse photoemission spectra reproduce very well the most recently measured spectra, as do also our theoretical optical spectra. In contrast to the normal situation, we find that the local density approximation to the density functional gives calculated equilibrium volumes in much better agreement with experiment than does the generalized gradient corrected functional. We also address the problem of treating relativistic $p$ electrons with methods based on a scalar-relativistic basis set and show that the effect is rather small for the present systems.
\end{abstract}

DOI: 10.1103/PhysRevB.66.035117

PACS number(s): 71.20.Nr, 71.15.Qe, 71.15.Rf, 71.15.Mb

\section{INTRODUCTION}

The cubic Hg II-VI systems $\beta$-HgS, HgSe, and $\mathrm{HgTe}$ are technologically interesting materials with application in quantum electronics. They form part of several artificial nanostructured materials. ${ }^{1-3}$

The electronic structure of $\mathrm{HgSe}$ has recently been the subject of debate, due to contradicting results regarding the ordering of the highest valence levels and possible existence of a band gap. The investigations involved include photoemission spectroscopy, ${ }^{4,5}$ magneto-optical Fourier transform spectroscopy, ${ }^{6}$ and theoretical calculations based on densityfunctional theory, both with and without quasiparticle corrections. ${ }^{7}$

The classical view of the electronic structure of $\mathrm{HgSe}$ is that it is a zero-gap semiconductor (i.e., a semimetal) with an inverted band structure compared to the isoelectronic systems $\mathrm{ZnSe}$ and $\mathrm{CdSe}$. The "inversion" consists of the following. The $\mathrm{Hg} s$ level, which forms a state of $\Gamma_{6}$ symmetry (with place for two electrons) at the zone center, has been pulled down below (at least) the $\Gamma_{8}$ level due to the large effective positive charge of the $\mathrm{Hg}$ core. The valence electrons therefore suffice to occupy only two of the four levels of $\Gamma_{8}$ character. The unoccupied $\Gamma_{8}$ levels become part of the conduction band, which consequently becomes degenerate with the uppermost valence band at $\Gamma$, creating a zero fundamental energy gap.

With the photoemission experiments reported in Ref. 4, this picture was put into question, suggesting a positive band gap in $\mathrm{HgSe}$.

Experimentally, the inverse photoemission intensities for these systems are very low in a region just above the Fermi level, so low that from the spectra itself it becomes difficult to distinguish between a situation with a band gap present or, alternatively, just a very low intensity due to large band dis- persion combined with small transition matrix elements. Therefore, we found it relevant to explicitly calculate the photoemission spectra, including the full relativistic transition matrix elements, of these compounds. The photoemission spectra of $\mathrm{HgSe}$ and $\mathrm{HgTe}$ have previously been compared only to a theoretical total density of states (DOS). ${ }^{5}$

Optical spectroscopy is a useful method for detecting metallic behavior, measuring band gaps, and making more general analyses of the electronic structure. For this reason, we found it relevant to explicitly calculate the optical spectra, using the full transition matrix elements, and compare with available experimental data. ${ }^{8,9}$ From a calculational point of view, no systematic study of these compounds has been previously performed with the spin-orbit coupling taken into account. Rohlfing and Louie ${ }^{7}$ calculated the band structure and DOS for HgSe using $G W$ (Ref. 10) and the local density approximation (LDA) with norm-conserving pseudopotentials and a basis set consisting of local Gaussian orbitals. Other studies have also been made, based on empirical or semiempirical methods. ${ }^{8,11,12}$

The II-VI mercury compounds have also caught our interest for a rather technical reason. Relativistic $p$ electrons are important in these systems. However, fully relativistic $p_{1 / 2}$ states are not zero at the origin, which leads to bad convergence when these states are to be expanded in basis functions derived from a scalar-relativistic Hamiltonian. This problem is very general and is present in all methods where scalar-relativistic basis functions are used. Nordström et al. ${ }^{13}$ have recently discussed this problem for actinides, where the effect is large due to the semicore $6 p$ states. In actinides, and also in lanthanides, the $p$ states have an additional problem not present in the systems we address. The spin-orbit coupling splits the semicore $p$ states into two well-separated peaks. Due to the way in which the basis set is constructed, these two peaks are described with the same linearization energy $E_{\nu}$ (see Sec. II), which is then chosen to lie in be- 
tween the two peaks. Such a linearization energy does not describe any of the peaks in an optimal way, which also leads to bad convergence of the basis set. In our case, the $6 p$ states form wide bands, and therefore this $E_{\nu}$ problem should not be present. Furthermore, in the method used here, the spinorbit coupling is neglected in the region of space outside the muffin-tin spheres.

The net results of all these shortcomings is that equilibrium properties such as volume and bulk modulus will depend on the radii of the muffin-tin spheres. We have measured how large the effect of changing the muffin-tin radii is by calculating the equilibrium volume and bulk modulus using both constant sphere radii and scaling them with the lattice parameter.

$\mathrm{HgSe}$ and $\mathrm{HgTe}$ crystallize in the zinc-blende structure, a rather open structure, at ambient pressure and temperature, with lattice parameters $6.08 \AA$ and $6.46 \AA$, respectively. In contrast, $\mathrm{HgS}$ in the zinc-blende structure, $\beta$ - $\mathrm{HgS}$, becomes stable only at moderately elevated temperatures, ${ }^{14}$ with lattice parameter $5.85 \AA$ A. $\beta$ - $\mathrm{HgS}$ can also be stabilized at ambient temperature through a couple of percent doping with a transition metal, e.g., $\mathrm{Fe},{ }^{15}$ and $\mathrm{CdS} / \mathrm{HgS} / \mathrm{CdS} /$ heterostructures. ${ }^{3}$

\section{METHOD}

In the band-structure calculations presented here, we have used the full-potential linear muffin-tin orbital (FP-LMTO) method. ${ }^{16,17}$ In this method, the Kohn-Sham equations ${ }^{18}$ are solved for a general potential without any shape approximation. Two different approximations to the density functional were used: The LDA based on the Monte Carlo data calculated by Ceperley and Alder ${ }^{19}$ and parametrized by Perdew and Zunger, ${ }^{20}$ and the generalized gradient approximation $(\mathrm{GGA})^{21}$

Our method is linear; i.e., the basis functions are constructed by expanding around fixed energies $E_{\nu}$. For $\mathrm{Hg}$, the $6 s, 6 p$, and $5 d$ orbitals were included in the basis set. For the chalcogens we included the $n s, n p$, and $n d$ orbitals, where $n=3,4$, and 5 for $\mathrm{S}, \mathrm{Se}$, and Te, respectively. Since the elements in these systems are heavy, spin-orbit coupling should be important, and it was therefore included in our calculations. In the FP-LMTO method, spin-orbit coupling is treated in a first variational step.

In the calculations of the photoemission and optical spectra, the experimental lattice parameters were used. The theoretical photoemission spectra were calculated using the fully relativistic formalism described in detail in Ref. 22. For explicit formulas, we refer to that paper. The method assumes a one-particle approach, and only electric dipole transitions are taken into account. Also, the local approximation is used; i.e., all contributions from terms including transitions between two different atomic sites are neglected.

The experimental photoemission spectra (PES), with which we compare our calculated spectra, were measured using the $\mathrm{He} \mathrm{I}$ and $\mathrm{Ar} I$ lines $(21.22 \mathrm{eV}$ and $11.70 \mathrm{eV}$, respectively) and the inverse PES (IPES) at the isochromat energy $9.5 \mathrm{eV}$, and these are also the energies we have assumed in our calculations. In order to simulate a finite lifetime of the excited states, we convoluted the spectra with a Lorentzian, whose width increases quadratically with the photon energy. Its full width at half maximum (FWHM) was $10 \mathrm{meV}$ at the photon energy $1 \mathrm{eV}$ for both the occupied and unoccupied spectra. The spectra were subsequently convoluted with a Gaussian of constant width, with FWHM=0.5 eV (to match the broadening used in Ref. 5) for the unoccupied DOS and IPES and FWHM $=0.1 \mathrm{eV}$ for the occupied DOS and PES.

The basic underlying theory we employ in our calculations of the optical spectra is linear response theory. ${ }^{23}$ For undoped samples, the intraband transitions should be negligible, and thus we have not included any intraband transitions in our calculated spectra. For doped samples, however, the intraband transitions may have important effects on the low-energy part of the spectra. The absorption and refractive index spectra of the doped samples in Ref. 8 are in fact governed by the intraband term. More details regarding the method used in the present optical calculations can be found in, e.g., Ref. 24.

The absorptive part of the optical conductivity was broadened with a Lorentzian, with a width increasing quadratically with the excitation energy and a FWHM of $10 \mathrm{meV}$ at the photon energy $1 \mathrm{eV}$. In addition, the spectra were convoluted with a Gaussian of constant FWHM $=0.1 \mathrm{eV}$, simulating instrumental broadening. The corresponding dispersive optical conductivity was then calculated by performing a KramersKronig transformation of the broadened absorptive part. ${ }^{25}$

\section{RESULTS}

\section{A. Ground-state properties}

As a basis for further analysis, we first briefly discuss the chemical bonding. In a simple chemical picture of the $\mathrm{Hg}$ II-VI semiconductors, Hg contributes with two $s$ electrons to the bonding and the chalcogen with two $s$ and four $p$ electrons. In the Hg II-VI compounds, the bonding is rather covalent and dominated by $s p^{3}$ hybrids, as indicated by the tetrahedral coordination. In comparison with the $\mathrm{Ca}, \mathrm{Sr}$, and $\mathrm{Ba}$ chalcogenides the ionicity of the $\mathrm{Hg}$ chalcogenides is reduced. The $\mathrm{Hg} d$ electrons are partially delocalized, and therefore the effective nuclear charge experienced by the valence electrons is increased. This causes more tightly bound $\mathrm{Hg}$ valence $s$ electrons and, hence, a less ionic and more covalent bond. In this respect, these systems are very similar to the isoelectronic $\mathrm{Cd}$ and $\mathrm{Zn} \mathrm{II-VI} \mathrm{semiconductors.} \mathrm{The}$ $d$-shell delocalization is stronger in $\mathrm{Hg}$ than in $\mathrm{Cd}$ or $\mathrm{Zn}$ and in fact so strong that it causes the $s$ level to be pulled down below the chalcogen $p$ level; i.e., an inverted band structure is formed. The role of the $d$ electrons in the bonding in II-VI semiconductors has been further discussed by Wei and Zunger. $^{26}$

Let us now compare this with the picture emerging from our calculations. Figure 1 shows the atom- and orbitalresolved DOS for $\mathrm{HgSe}$ at the experimental volume. The uppermost panel shows the $s$ states for both $\mathrm{Hg}$ and $\mathrm{Se}$, the middle panel $p$ states, and the lowermost panel $d$ states. The solid lines are Hg DOS, and the dotted lines are Se DOS. All panels have the same scale in order to facilitate comparison. 


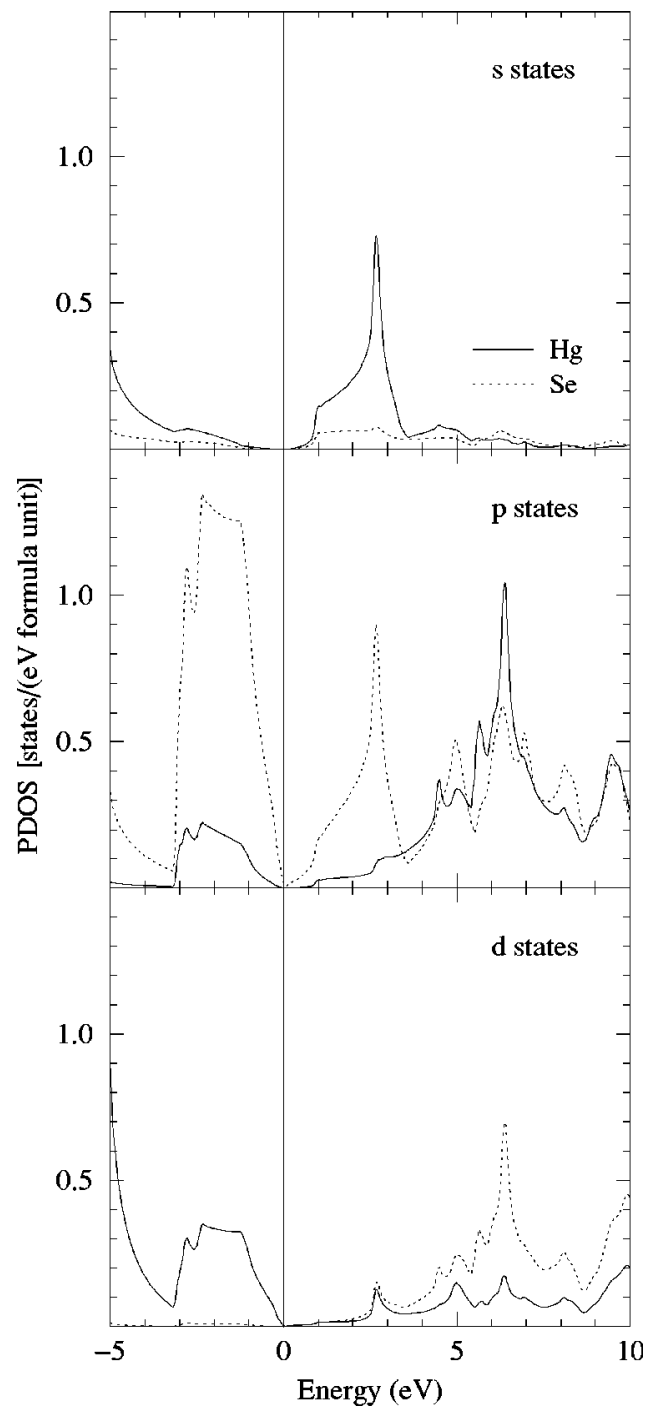

FIG. 1. Atom- and orbital-resolved DOS for $\mathrm{HgSe}$ at the experimental volume. The Fermi level is at zero.

For $\beta-\mathrm{HgS}$ and $\mathrm{HgTe}$ the main features are the same as for $\mathrm{HgSe}$ and the discussion here is valid also for those systems.

From the two lowest panels of Fig. 1 we see that most of the $\mathrm{Hg} d$ states are concentrated below $-5 \mathrm{eV}$, but that there is a significant $p d$ hybridization in the region $0-5 \mathrm{eV}$ below the Fermi level. Further, judging from the orbitalresolved occupation numbers inside the muffin-tin spheres, about one $\mathrm{Hg} d$ electron is delocalized into the interstitial region. Thus, the picture of a partly delocalized $\mathrm{Hg} d$ shell is confirmed.

In Fig. 2 we show the LDA band structures calculated at the experimental equilibrium volumes for all three systems along the $\Lambda$ and $\Delta$ directions of the Brillouin Zone (BZ) in the energy range from -10 to $10 \mathrm{eV}$. For $\mathrm{HgSe}$, we have also plotted bands calculated using the GGA (dashed lines), for comparison.

Let us first concentrate on the $\mathrm{HgSe}$ band structure and compare it with the calculations in Ref. 7. Clearly, our LDA bands are very similar to the ones shown in Ref. 7. The main difference is that the third band counting from the valence-

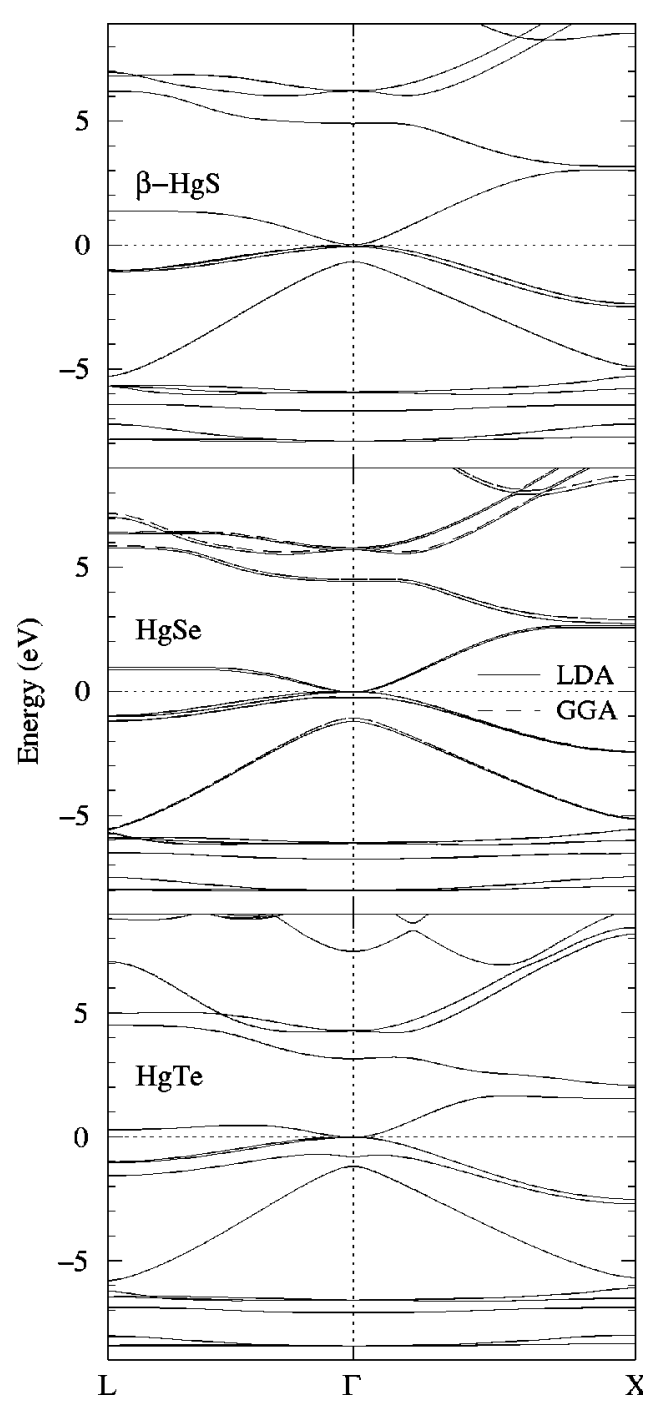

FIG. 2. Band structures along $\Lambda$ and $\Delta$ for $\beta-\mathrm{HgS}, \mathrm{HgSe}$, and $\mathrm{HgTe}$. For HgSe, GGA results (dashed lines) are also included for comparison. The Fermi level (or VBM for $\beta-\mathrm{HgS}$ ) is at zero.

band maximum is around $1 \mathrm{eV}$ higher in energy in the $G W$ calculation and is thus much closer to the second highest valence band. The differences between our LDA band structure and the LDA band structure in Ref. 7 give an estimate of the uncertainty, originating from details of the method used (e.g., linearization, basis set used, construction of pseudopotentials). We find that the band structures are very similar overall up to around $8 \mathrm{eV}$ above the Fermi energy. Above this energy, both the ordering of the bands and the absolute energy position of the bands differ.

For $\mathrm{HgSe}$, the band structure from the GGA calculation is also plotted. Evidently, the differences between the LDA and GGA eigenvalue spectra are tiny. This is not surprising, and in general, we expect the same type of conclusion to be true for any (bulk) system. From this, we also conclude that the choice of functional in the calculation of the excitation spectra will not greatly affect the results, and thus we have chosen to calculate the photoemission spectra and the optical spectra using LDA only. 


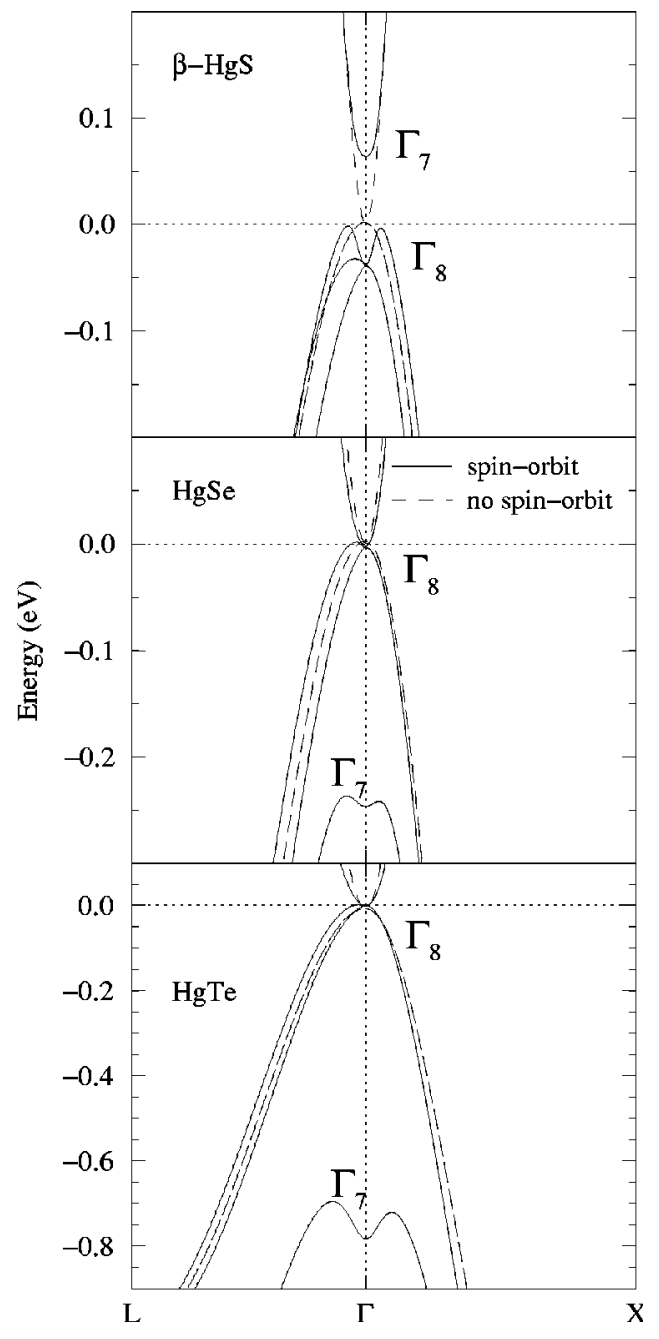

FIG. 3. Detailed plots around the Fermi level of the band structures along $\Lambda$ and $\Delta$ for $\beta-\mathrm{HgS}, \mathrm{HgSe}$, and $\mathrm{HgTe}$, both with and without spin-orbit coupling (solid and dashed lines, respectively). The representations $\Gamma_{7}$ and $\Gamma_{8}$ refer to the symmetry at the $\Gamma$ point of the solid bands. The Fermi level (or VBM for $\beta-\mathrm{HgS}$ ) is at zero.

The main difference between the band structures for $\beta-\mathrm{HgS}, \mathrm{HgSe}$, and $\mathrm{HgTe}$ in the region shown is the size of the gap between the two highest valence bands. This gap is largest for $\mathrm{HgTe}$, where it is of the order of $1 \mathrm{eV}$, but appears to be virtually zero for $\beta-\mathrm{HgS}$. This clear trend makes it easy to associate this gap with the spin-orbit splitting of the $p$ chalcogen states. This, however, turns out to be an oversimplification, which will be discussed in the next paragraph where the ordering of the valence levels close to the Fermi level is discussed in greater detail.

In Fig. 3, the bands around the Fermi level are shown in detail. The solid lines represent bands calculated with spinorbit coupling included, and the dashed lines are the bands calculated without spin-orbit coupling, but with all other details of the calculation unchanged. Without spin-orbit coupling, the bands form a three fold-degenerate level (with place for six electrons since spin is not counted in the degeneracy of the simple group) with $\Gamma_{15}$ symmetry. In $\mathrm{Cd}$ and $\mathrm{Zn}$ II-VI semiconductors, the corresponding levels are all filled, whereas in the Hg II-VI systems, one of these levels remains
TABLE I. Theoretical equilibrium volumes calculated using two different functionals [LDA (Refs. 19 and 20) and GGA (Ref. 21)] and two different schemes for the muffin-tin radii ("scaled" and "constant"), compared with experimental equilibrium volumes (Ref. 28).

\begin{tabular}{lccccc}
\hline \hline \multirow{2}{*}{ System } & $V_{\text {expt }}(\AA)$ & Scaled & Constant & Scaled & Constant \\
\hline$\beta-\mathrm{HgS}$ & 50.1 & 50.1 & 49.5 & 54.8 & 54.0 \\
$\mathrm{HgSe}$ & 56.2 & 56.9 & 56.1 & 62.6 & 61.4 \\
$\mathrm{HgTe}$ & 67.4 & 68.8 & 67.1 & 75.8 & 73.8 \\
\hline \hline
\end{tabular}

unoccupied since the electrostatically pulled-down $s$ level has to be filled first. This means that the highest valence band and lowest conduction band become degenerate in one point $\Gamma$, and all three systems are thus semimetals when spin-orbit coupling is neglected. Away from $\Gamma$ along the $\Lambda$ and $\Delta$ directions, the threefold level splits up into a onefold conduction band and a twofold valence band.

If the spin-orbit coupling is included, the double-group representation must be used, and $\Gamma_{15}$ splits up into two levels: $\Gamma_{8}$, which can accommodate four electrons, and $\Gamma_{7}$, with place for two electrons. Both these levels contain both $p$ as well as $d$ character. In both $\mathrm{HgSe}$ and $\mathrm{HgTe}, \Gamma_{8}$ is higher in energy than $\Gamma_{7}$, whereas the situation is reversed in $\beta$ - $\mathrm{HgS}$. The reason is the following. The energy difference between the $\Gamma_{8}$ and $\Gamma_{7}$ levels is determined by the chalcogen $p$ spinorbit splitting, the $\mathrm{Hg} d$ spin-orbit splitting, and the strength of the coupling between these states, the $p d$ coupling. For the $p$ states, the $\Gamma_{8}$ symmetry is higher in energy than the $\Gamma_{7}$, whereas the situation is reversed for the $d$ states. Thus, if the $p$ spin-orbit coupling becomes sufficiently small (like in sulphur), the order of the $\mathrm{Hg} d$ spin-orbit split states decides the order of the $\Gamma_{8}$ and $\Gamma_{7}$ levels. Alternatively, if the $d$ character becomes dominant in these bands due to large $p d$ coupling, the $\Gamma_{7}$ level might also end up higher than the $\Gamma_{8}$ level. With the order of the $\Gamma_{7}$ and $\Gamma_{8}$ levels reversed, a gap opens up in $\beta-\mathrm{HgS}$. This situation is further discussed in Ref. 27.

Finally, we also present some results for the equilibrium volumes and bulk moduli. These calculations were performed in four different ways, by combining the two alternatives for the functional (LDA or GGA) with the two alternative treatments of the muffin-tin spheres (muffin-tin radius scaled with lattice parameter or kept constant). The main reasons for this our interest are, first, a recent debate regarding how systems with large spin-orbit coupling in the valence or semicore states should be treated within fullpotential methods ${ }^{13}$ and, second, the fact that we observed that the systems under consideration here seemed to be an exception to the rule of thumb that the GGA, when combined with a full-potential method, usually gives volumes and bulk moduli in closer agreement with experiment than does the LDA.

In Table I, we compare our calculated equilibrium volumes with experimental data. ${ }^{28}$ The theoretical volumes and bulk moduli were extracted from a set of energy-volume points by fitting to the universal equation of state. ${ }^{29} \mathrm{We}$ see that the LDA results are very close to the experimental vol- 
TABLE II. Theoretical bulk moduli evaluated at the experimental volume, using two different functionals [LDA (Refs. 19 and 20) and GGA (Ref. 21)] and two different schemes for the muffin-tin radii ("scaled" and "constant"), compared with experimental data (Ref. 30).

\begin{tabular}{lccccc}
\hline \hline & & \multicolumn{2}{c}{$B_{\mathrm{LDA}}(\mathrm{GPa})$} & \multicolumn{2}{c}{$B_{\mathrm{GGA}}(\mathrm{GPa})$} \\
System & $B_{\text {expt }}(\mathrm{GPa})$ & Scaled & Constant & Scaled & Constant \\
\hline$\beta$-HgS & 68.6 & 67.0 & 66.4 & 65.5 & 64.9 \\
$\mathrm{HgSe}$ & 57.6 & 59.1 & 59.2 & 58.1 & 58.8 \\
$\mathrm{HgTe}$ & 46.7 & 47.8 & 47.8 & 46.4 & 46.7 \\
\hline \hline
\end{tabular}

umes with the constant-sphere calculations giving volumes on the average $1 \%$ less and the scaled-sphere calculations $1 \%$ larger than experiment. The GGA calculations give, on the average, $9 \%$ larger volumes than the LDA. Thus, in this case, the GGA performs much worse than the LDA. In contrast, the difference between the two alternative treatments of the muffin-tin spheres is rather small.

Table II contains calculated bulk moduli, evaluated at the experimental volume, compared with experimental data. ${ }^{30}$ Since the theoretical bulk modulus and volume strongly covary (an overestimated volume will almost certainly result in an underestimated bulk modulus), we have chosen to remove this dependence by evaluating the bulk modulus at the experimental volume. In this way, any trend in the bulk modulus that can be extracted from our calculations can be attributed to the second derivative of the energy-volume curve. We find that with this approach, there is no big difference between the LDA and GGA results for the bulk moduli. Regarding the effect of scaled or constant spheres on the bulk modulus, there is no clear trend and the average difference is small, always less than $2 \%$. Thus, the bulk modulus is not sensitive neither to the choice of functional nor to the treatment of the muffin-tin spheres for these systems. Our calculated bulk moduli agree extremely well with the experimentally measured ones.

\section{B. Photoemission spectra}

Figure 4 shows calculated DOS (dashed lines) and (I)PES (solid lines) for $\beta$-HgS, HgSe, and $\mathrm{HgTe}$ together with experimental (I) PES (Refs. 5 and 31) (solid circles). The $\beta$-HgS crystal, on which the photoemission measurements were performed, was doped with $4 \%$ Fe. For all three systems, our calculated DOS and IPES have been shifted upwards $0.7 \mathrm{eV}$ in order to align the dominant peaks in the experimental and calculated spectra. This offset in our calculated spectra (it is present also in the optical spectra) is due to the neglect of final-state effects in the calculations. For $\mathrm{HgSe}$, we have also plotted the unbroadened total DOS for the unoccupied states (long-dashed line) and unbroadened $G W$ DOS (Ref. 7) (dotted line).

The experimental HgSe and HgTe PES contain the following structures. There is a smaller peak around $-3 \mathrm{eV}$, a larger broader feature (which is much broader in HgSe than in $\mathrm{HgTe}$ ) centered around $-2 \mathrm{eV}$. Comparing with the partial DOS in Fig. 1, we see that mainly the chalcogen $p$ states

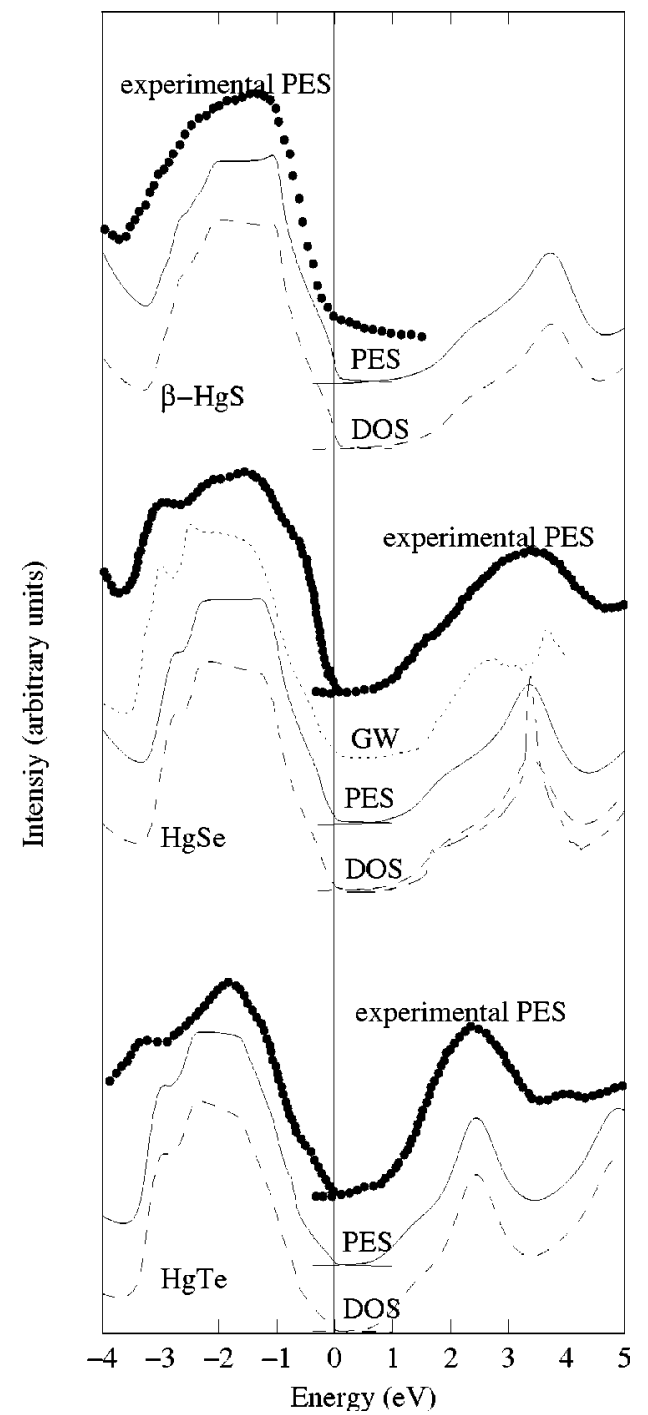

FIG. 4. Calculated DOS and PES for $\beta$-HgS, $\mathrm{HgSe}$, and $\mathrm{HgTe}$ compared with experimental PES and IPES from Ref. $31(\beta-\mathrm{HgS})$ and Ref. 5 (HgSe and HgTe). For $\mathrm{HgSe}$, the GW DOS from Ref. 7 is also included. Our calculated spectra for the unoccupied states have been shifted $0.7 \mathrm{eV}$ higher in energy in order to align the main peaks of the experimental and calculated spectra. The designation "PES" in the figure stands for both the PES and IPES spectra.

are responsible for these structures. The energy positions of these peaks are the same for both $\mathrm{HgSe}$ and HgTe. The spectra above the Fermi energy are dominated by one rather broad feature, whose position is strongly dependent on the chalcogen. This structure is, again, due to chalcogen $p$ states but also $\mathrm{Hg} s$ states. The experimental PES for $\beta-\mathrm{HgS}$ exhibits the same broad peak centered around $-2 \mathrm{eV}$, but lacks the structure at $-3 \mathrm{eV}$.

Both our calculated DOS and (I) PES reproduce the structures in the experimental spectra, as well as their relative amplitudes. We see that the effect of including the matrix elements is rather faint. The most prominent effect is that the peak around $-3 \mathrm{eV}$ becomes flatter. The $G W$ DOS is very similar to the LDA DOS below the Fermi energy. Above the Fermi energy, however, the $G W$ DOS exhibits a double-peak 


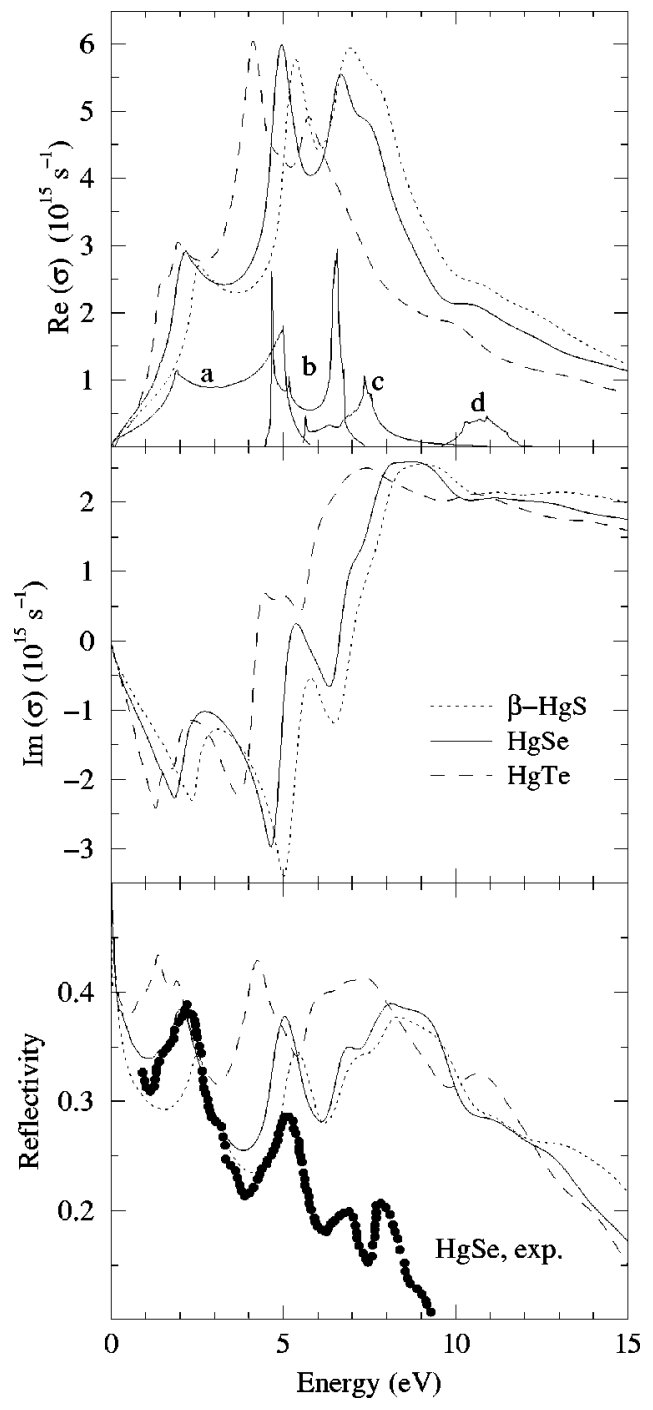

FIG. 5. Calculated optical conductivities and reflectivities for $\beta$-HgS, HgSe, and HgTe. The spectra (a)-(d) (multiplied by a factor of 2 for visibility) in the top panel are some representative band-resolved contributions to the absorptive optical conductivity of $\mathrm{HgSe}$. An experimental reflectivity spectrum for $\mathrm{HgSe}$ is also included (Ref. 9)

structure which is present neither in the experimental spectrum nor in the LDA DOS. In fact, the unbroadened LDA DOS has only one-and sharp-peak in that energy region. In our calculated spectra, also when the transition matrix elements are included, we find no evidence of the additional structure close to the Fermi energy reported in Ref. 4. Instead, our results are in accordance with several other experimental observations. $5,6,31,32$

\section{Optical spectra}

The optical conductivities and reflectivities for $\beta-\mathrm{HgS}$ (dotted lines), $\mathrm{HgSe}$ (solid lines), and HgTe (dashed lines) are plotted in Fig. 5.

In the top panel, the absorptive optical conductivities are shown. For $\mathrm{HgSe}$, we also give some representative bandresolved contributions [spectra (a)-(d)], which we now look closer at in order to resolve which bands contribute most to the different structures. In the energy region shown, there are five main peaks or structures (for $\mathrm{HgSe}$ at the energies 2, 5, 7,8 , and $11 \mathrm{eV}$ ). If the contributions from all individual pairs of bands are plotted individually, it is seen that the most important band-pair contributions can all be sorted into four distinct types, illustrated by the spectra (a)-(d).

The spectra of type (a)-(d) all involve the two highest valence bands (HVB's), but different conduction bands. Numbering the conduction bands so that the lowest conduction band (LCB) is band 1, spectra of type (a) involve conduction bands 1 and 2, type (b) involve conduction bands 3 and 4, type (c) conduction bands 5 and 6, and type (d) conduction bands 9 and 10 .

We have chosen to analyze the spectra here by resolving them into band contributions. The spectra can also be broken down into contributions from different regions and highsymmetry points in the BZ. Such an association of the main peaks with the high-symmetry points can be found in Ref. 9 . Another interesting analysis is to look at the dominant character $\left(s, p_{x}\right.$, etc.) of the valence and conduction bands involved. In a strict sense, such an analysis, however, is only possible inside the muffin-tin spheres, since the character of an eigenstate is undefined in the interstitial region. Moreover, a Se $p$ state, for example, expanded around the $\mathrm{Hg}$ muffin-tin sphere origin (transitions occur locally in our model) no longer has only $p$ character, and thus designations of the type "Se $p \rightarrow \mathrm{Hg} d$ transition" are ambiguous.

It is more meaningful to start from the perspective of the atoms. Then, the square-shaped DOS $0-3 \mathrm{eV}$ below the Fermi energy as well as the peaked structure around $2 \mathrm{eV}$ above the Fermi energy should be called chalcogen $p$ states, since these two structures originate from the four occupied and two unoccupied $p$ orbitals in the chalcogen atom, and the broadband peaking around $6 \mathrm{eV}$ above the Fermi energy should be thought of as $\mathrm{Hg} p$ states. With this designation, the low-energy part of the optical spectrum can in fact be characterized as (mainly) chalcogen $p \rightarrow p$ transitions and the high-energy part as (mainly) chalcogen $p \rightarrow \mathrm{Hg} p$ transitions. (Note that we are still strictly employing the dipole approximation.)

Panel 2 of Fig. 5 contains the dispersive parts of the optical conductivities. We have included these spectra in order to give a complete characterization of our calculated optical conductivities, so that any spectrum (absorption, refractive index, energy loss, dielectric function, etc.) can be calculated from our data for future comparison with experimental measurements.

Finally, the lowest panel of Fig. 5 shows the calculated reflectivities, which we have included since it is the most commonly measured optical property of a material. In the visible spectrum, we predict a reflectivity of around 35\%$40 \%$ for all three systems. Any measured reflectivity is likely to be lower, due to scattering. An experimental spectrum for $\mathrm{HgSe}$, measured by Guziewicz et al., ${ }^{9}$ is included in the lowest panel of Fig. 5. To facilitate comparison of the structures in the experimental and calculated spectra, the amplitude of the measured spectrum has been multiplied by a factor of 1.6 , so that the amplitudes of the experimental and theoreti- 
cal reflectivity spectra become equal at the first peak. Furthermore, just as for the IPES, the calculated spectra underestimate the peak energy positions. Therefore, the experimental spectrum has been moved down $0.7 \mathrm{eV}$ in energy. Apparently, the amplitude in the experimental spectrum is much lower than the calculated one, and it also decreases more quickly with energy, whereas the relative peak positions are in good agreement.

Finally, we also compare our optical calculations with another recent reflectivity measurement. Einfeldt et al. ${ }^{8}$ measured, for $\mathrm{HgSe}$, the reflectivity of the first peak in the reflectivity spectrum, which is situated close to $2 \mathrm{eV}$ in our calculated spectrum and $0.7 \mathrm{eV}$ higher in the experimental spectrum. We find a reflectivity of $38 \%$ for this peak. In the experiment, this peak has $29 \%$ reflectivity at $5 \mathrm{~K}$ and $35 \%$ reflectivity at room temperature.

We now move to a brief discussion of trends in the spectra associated with changing the chalcogen. The spectra for all three systems are quite similar, with the most obvious difference that the peaks move to lower energies the heavier the chalcogen is. The same effect can be seen in the optical spectra for lead chalcogenides. ${ }^{33}$ The differences between the three systems are mainly different lattice parameters (larger the heavier the chalcogen) and different number of nodes on the wave functions (more nodes the heavier the chalcogen) in the valence orbitals. These two differences should partly cancel each other, when it comes to the energies of the main peaks. The more nodes an orbital has, the wider is the band. On the other hand, the larger the lattice parameter, the more contracted are the bands. We tested the effect of changing the lattice parameter by recalculating the spectra of $\beta$ - $\mathrm{HgS}$ and $\mathrm{HgTe}$ using the $\mathrm{HgSe}$ experimental lattice parameter. As expected, the three spectra become much more similar. The main peaks are now almost on top of each other (not shown). However, the spectrum for HgTe stands out, with some structures not present in the other spectra, mostly due to the strong spin-orbit coupling in this compound. The peaks in $\mathrm{HgTe}$ are generally also more smeared out, which is consistent with the picture that the bands broaden the more nodes the orbitals contain.

\section{DISCUSSION AND CONCLUSIONS}

Regarding the bonding and ground-state properties in the mercury II-VI systems, our main conclusions are the following. The bonding is covalent with $s p^{3}$-hybrid formation, due to the partly delocalized $\mathrm{Hg} d$ electrons. This delocalization also causes the $\Gamma_{6}$ level to sink down below the Fermi level and is thus a prerequisite for the semimetallic behavior found in $\mathrm{HgSe}$ and $\mathrm{HgTe}$. We find the order $\Gamma_{8}, \Gamma_{7}$, and $\Gamma_{6}$ counting from the uppermost valence band for $\mathrm{HgSe}$ and $\mathrm{HgTe}$. This is the same ordering reported for $\mathrm{HgSe}$ by Rohlfing and Louie, ${ }^{7}$ but contradicts the experiments reported by Orlowski et $a .^{32}$ (for $\mathrm{HgTe}$ ) and von Truchsess et al. ${ }^{6}$ (for $\mathrm{HgSe}$ ).
However, the conclusion regarding the ordering of the valence levels in HgSe in Ref. 6 is based on the assumption that electric-dipole transitions are symmetry forbidden between the $\Gamma_{8}$ and $\Gamma_{7}$ levels. But since both levels contain both $d$ and $p$ states, such transitions are in fact allowed and strong. In $\beta$-HgS, we find the $\Gamma_{7}$ level above the $\Gamma_{8}$ level. Both these levels are well above the $\Gamma_{6}$ level. Because of the difference in degeneracy, this reordering opens a gap around $\Gamma$ in $\beta$-HgS. In $\mathrm{HgTe}$, the $\Gamma_{6}$ and $\Gamma_{7}$ levels are very close together in our calculation, and the energy difference would decrease further with quasiparticle corrections included. It is quite plausible that such corrections could alter the order of these levels. This would explain the recent angle-resolved photoemission spectra measured by Orlowski et al. ${ }^{32}$

We find that our LDA-calculated volumes agree with the experimental equilibrium volumes to within $1 \%$. The GGA gives much worse results, which is quite unusual.

Moving on to our muffin-tin sphere test, we find that our calculation using constant muffin-tin spheres gives $2 \%$ smaller volumes than if the muffin-tin spheres are maximized at each individual energy-volume point. For thorium, ${ }^{13}$ the corresponding difference is close to $10 \%$. We conclude that the much larger effect in thorium is associated with the " $E_{\nu}$ problem" for semicore states explained in the Introduction and also with the fact that the spin-orbit coupling for the valence electrons in thorium (a $5 f$ system) is larger than in the systems addressed here.

The experimental PES, IPES, and optical spectra are very well described by our calculations. The relative peak positions and amplitudes as well as the overall structure of the spectra are all well reproduced. The $G W$ calculation $^{7}$ for $\mathrm{HgSe}$ IPES gives an average peak position in closer agreement with the experimental spectrum than do our calculations. However, the $G W$ DOS shows a clear double-peak structure, not present in the experimental spectrum. We also conclude that the effect of the transition matrix elements on the PES and IPES is small. An explanation for this is that, in general, the transition probabilities should be more important in systems where one partial DOS does not dominate over all the other. The role of the transition matrix elements is to modulate the partial DOS contributions, with different modulation amplitudes for different $l$ and different atoms. In the present case, the chalcogen $p$ channel dominates both the PES and IPES spectra, and therefore the relative effect of modulation is limited.

\section{ACKNOWLEDGMENTS}

This work was financed by the Swedish Foundation for International Cooperation in Research and Higher Education (STINT) and the Swedish Council for Natural Sciences (NFR). Discussions with P. Korzhavyi and C. Persson are acknowledged, and J. M. Wills is acknowledged for making the FP-LMTO code available. 
${ }^{1}$ H. Wissmann, T. Tran-Anh, S. Rogaschewski, and M. von Ortenberg, J. Cryst. Growth 201-202, 619 (1999).

${ }^{2}$ S.V. Kershaw, M. Harrison, A.L. Rogach, and A. Kornowski, IEEE J. Sel. Top. Quantum Electron. 6, 543 (2000).

${ }^{3}$ A. Mews, A.V. Kadavanich, U. Banin, and A.P. Alivisatos, Phys. Rev. B 53, R13 242 (1996).

${ }^{4}$ K.U. Gawlik et al., Phys. Rev. Lett. 78, 3165 (1997).

${ }^{5}$ D. Eich et al., Phys. Rev. B 61, 12666 (2000).

${ }^{6}$ M. von Truchsess et al., Phys. Rev. B 61, 1666 (2000).

${ }^{7}$ M. Rohlfing and S.G. Louie, Phys. Rev. B 57, R9392 (1998).

${ }^{8}$ S. Einfeldt et al., Phys. Rev. B 51, 4915 (1995).

${ }^{9}$ E. Guziewicz et al., Phys. Rev. B 55, 4405 (1997).

${ }^{10}$ L. Hedin, Phys. Rev. 139, A796 (1965); L. Hedin and S. Lundqvist, in Solid State Physics, Advances in Research and Application, edited by F. Seitz, D. Turnbull, and H. Ehrenreich (Academic, New York, 1969), Vol. 23, p. 1.

${ }^{11}$ S. Bloom and T.K. Bergstresser, Phys. Status Solidi 42, 191 (1970).

${ }^{12}$ S.N. Ekpenuma and C.W. Myles, J. Phys. Chem. Solids 51, 93 (1990).

${ }^{13}$ L. Nordström et al., Phys. Rev. B 63, 35103 (2001).

${ }^{14}$ G.G. Roberts, E.L. Lind, and E.A. Daris, J. Phys. Chem. Solids 30, 833 (1969).

${ }^{15}$ K. Dybko, W. Szuszkiewicz, and B. Witoska, Defect Diffus. Forum 121/122, 41 (1995).

${ }^{16}$ J. M. Wills, O. Eriksson, M. Alouani, and O. L. Price, in Electronic Structure and Physical Properties of Solids, edited by H. Dreyssé (Springer, Berlin, 2000).
${ }^{17}$ O.K. Andersen, Phys. Rev. B 12, 3060 (1975); H. L. Skriver, The LMTO Method (Springer, Berlin, 1984).

${ }^{18}$ P. Hohenberg and W. Kohn, Phys. Rev. 136, B864 (1964); W. Kohn and L.J. Sham, Phys. Rev. 140, A1133 (1965).

${ }^{19}$ D.M. Ceperley and B.J. Alder, Phys. Rev. Lett. 45, 566 (1980).

${ }^{20}$ J.P. Perdew and A. Zunger, Phys. Rev. B 23, 5048 (1981).

${ }^{21}$ J. P. Perdew, in Electronic Structure of Solids 1991, edited by P. Ziesche and H. Eschrig (Akademie Verlag, Berlin, 1991).

${ }^{22}$ P. Marksteiner et al., Phys. Rev. B 34, 6730 (1986).

${ }^{23}$ R. Kubo, J. Phys. Soc. Jpn. 12, 570 (1957); C.S. Wang and J. Callaway, Phys. Rev. B 9, 4897 (1974).

${ }^{24}$ M. Alouani and J.M. Wills, Phys. Rev. B 54, 2480 (1996); A. Delin et al., ibid. 60, 14105 (1999).

${ }^{25}$ A. Delin, Opt. Commun. 167, 105 (1999).

${ }^{26}$ S.H. Wei and A. Zunger, Phys. Rev. B 37, 8958 (1988).

${ }^{27}$ A. Delin, Phys. Rev. B 65, 153205 (2002).

${ }^{28}$ P. Villars and L. D. Calvert, Pearson's Handbook of Crystallographic Data for Intermetallic Phases, 2nd Edition (ASM International, Metals Park, OH, 1985).

${ }^{29}$ P. Vinet, J.H. Rose, J. Ferrante, and J.R. Smith, J. Phys.: Condens. Matter 1, 1941 (1989).

${ }^{30}$ K. Kumazaki, Phys. Status Solidi A 33, 615 (1976).

${ }^{31}$ B.J. Kowalski et al., J. Electron Spectrosc. Relat. Phenom. 85, 17 (1997).

${ }^{32}$ N. Orlowski et al., Phys. Rev. B 61, R5058 (2000).

${ }^{33}$ A. Delin, P. Ravindran, O. Eriksson, and J.M. Wills, Int. J. Quantum Chem. 69, 349 (1998). 